\title{
Avaliação Neuropsicológica das Funções Executivas: Considerações Metodológicas
}

\author{
Neuropsychological Assessment of Executive Functions: \\ Methodological Questions
}

\author{
Amer Cavalheiro Hamdan* \& Ana Paula de Almeida Pereira \\ Universidade Federal do Paraná
}

\begin{abstract}
Resumo
$\mathrm{O}$ interesse pelo estudo das funções executivas tem crescido nos últimos anos. O objetivo deste artigo é revisar algumas questões metodológicas e conceituais relacionadas à avaliação neuropsicológica das funções executivas. As principais limitações e dicotomias relacionadas às funções executivas são apresentadas e a importância de um modelo teórico que fundamente os instrumentos de avaliação e suas interpretações clinicas analisada. Conclui-se que as evidências provenientes da investigação das funções executivas ainda não permitem a compreensão satisfatória desses processos. Sugere-se, a adoção de um modelo específico para o estudo das funções executivas.

Palavras-chave: Neuropsicologia; Testes neuropsicológicos; Lobo frontal.

Abstract

The article aims to review methodological and conceptual issues related to neuropsychological assessment of executive functions. The main limitations and dichotomies of current studies related to executive functions are presented and the importance of theoretical support to validate the instruments and their clinical interpretation are discussed. There is still the need of additional evidences on executive functions that allow a complete understanding of all the processes involved. Finally, it is suggested the adoption of a specific model that may guide the study of the executive functions.

Keywords: Neuropsychology; Neuropsychological tests; Frontal lobe.
\end{abstract}

A avaliação neuropsicológica das funções executivas (FE) tem sido objeto de interesse crescente de pesquisadores e do público em geral. Esta afirmação é evidenciada por um crescente número de publicações (artigos e livros), bem como por encontros e debates dedicados a esse tema. Revisões recentes publicadas em periódicos internacionais (Faw, 2003; Funahashi, 2001; Royall et al., 2002; Stuss \& Levine, 2002; Tirapu-Ustárroz, MuñozCéspedes, \& Pelegrín-Valero, 2002) atestam esse interesse. Congressos e encontros têm sido promovidos exclusivamente para debater as FE (Grafman, Holyoak, \& Boller, 1995). Livros, obras de referência e capítulos de livros, publicados recentemente, demonstram também o crescente interesse por esse assunto (Fogel, Schiffer, \& Rao, 2000; Gazzaniga, Irvy, \& Mangun, 1998; Spreen \& Strauss, 1998). Para o público leigo, livros de divulgação foram publicados (Damásio, 1996; Goldberg, 2002). Entretanto, apesar do grande interesse de pesquisadores e do público em geral, permanecem ainda muitas dúvidas sobre o conceito do que é ou são as FE.

O dicionário da International Neuropsychological Society define funções executivas como "as habilidades

\footnotetext{
${ }^{*}$ Endereço para correspondência: Rua Paulo Friebe, 265, Bairro Alto, Curitiba, PR, Brasil, CEP 82820-420. Tel. (41) 3367 3011.E-mail: achamdan@ufpr.br
}

cognitivas necessárias para realizar comportamentos complexos dirigidos para determinado objetivo e a capacidade adaptativa as diversas demandas e mudanças ambientais" (Loring, 1999, p. 64). Tradicionalmente, FE referem-se às habilidades cognitivas envolvidas no planejamento, iniciação, seguimento e monitoramento de comportamentos complexos dirigidos a um fim. Na avaliação neuropsicológica, a denominação FE é utilizada para designar uma ampla variedade de funções cognitivas que implicam: atenção, concentração, seletividade de estímulos, capacidade de abstração, planejamento, flexibilidade de controle mental, autocontrole e memória operacional (Green, 2000; Loring, 1999; Spreen \& Strauss, 1998). Inúmeros testes e baterias neuropsicológicas têm sido empregados para avaliar as FE (Green, 2000; Spreen \& Strauss, 1998). Déficits nas FE tem sido relacionadas a alterações dos lobos frontais.

Contudo, dúvidas metodológicas e conceituais sobre o que são as FE persistem (Royall et al., 2002; Stuss \& Alexander, 2000). A simples constatação da presença de alterações cognitivas comportamentais relacionadas à área frontal não é suficiente para explicar o funcionamento complexo das FE. Algumas questões têm sido levantadas, tais como: Quais são os instrumentos empregados na avaliação neuropsicológica das FE? As FE são coordenadas por uma única função ou atuam como 
módulos independentes? O presente artigo tem por objetivo revisar algumas questões metodológicas e conceituais relacionadas à avaliação neuropsicológica das funções executivas.

\section{Breve Histórico das Funções Executivas}

A avaliação neuropsicológica das FE teve início com a observação de pacientes com lesões frontais e alterações comportamentais subseqüentes a essas lesões. No século XIX, surgiram as primeiras explicações que relacionaram as lesões dos lobos frontais às alterações do comportamento executivo. No século XIX, os frenologistas Gall e Spurzheimer suspeitaram que os lobos frontais poderiam ser responsáveis pela fala e cálculo (EstévezGonzáles, Garcia-Sánchez, \& Barraquer-Boras, 2000). Ainda nesse período, Broca descreveu diversos casos de afasia relacionados a lesões do lobo frontal esquerdo (Freinberg \& Farah, 1997).

No século XIX, Harlow descreveu as alterações comportamentais de Phineas Gage (Gazzaniga et al., 1998). O caso de Phineas Gage foi a primeira tentativa de descrição detalhada do comprometimento das FE decorrente de uma lesão dos lobos frontais. Em 1848, Gage trabalhava como engenheiro ferroviário em Nova Inglaterra, nos EUA. Sua função consistia em supervisionar o trabalho de construção de uma estrada de ferro. Entre suas obrigações, estava a de colocar explosivos em buracos e detoná-los para abrir caminho para a colocação de novos trilhos. Gage utilizava uma barra de ferro para apertar a pólvora. Por acidente, ele detonou o explosivo e a barra de ferro atravessou o seu crânio, ocasionando um grave acidente. Para surpresa de todos, Gage não morreu e tampouco perdeu a consciência. $\mathrm{O}$ calor da barra de ferro cauterizou a ferida em seu cérebro. Apesar de ter sobrevivido a este grave acidente, Gage sofreu mudanças profundas. De um empregado exemplar, passou a ser irresponsável e com comportamento socialmente inadequado. John Harlow, médico que o acompanhou, aventou que Gage havia sofrido lesões nos lobos frontais e que essa região é responsável por planejamentos e a execução de comportamentos socialmente adequados. Damásio e colegas (Gazzaniga et al., 1998), utilizando técnicas da neuroimagem do crânio de Gage, reconstruíram a trajetória da barra de ferro e concluíram que ela lesionou áreas do lobo frontal esquerdo e direito, em particular as áreas órbito-frontal e anterior-medial, e que estas regiões estão relacionadas ao comportamento socialmente adequado.

A moderna era da avaliação neuropsicológica das FE teve início com os trabalhos pioneiros de Luria (TirapuUstárroz et al., 2002). Mediante estudos com pacientes lesionados, advindos de ferimentos da Segunda Guerra Mundial, Luria construiu um modelo explicativo para as lesões dos lobos frontais. Para Luria (1981), o lobo frontal centraliza a responsabilidade por planejamentos, pro- gramação, regulação e verificação do comportamento intencional. Posteriormente, Lezak (1995; TirapuUstárroz et al., 2002) desenvolveu as idéias originais de Luria, cunhou o termo FE, dividindo-a em quatro categorias: (a) formulação de metas; (b) planejamento; (c) realização de planos dirigidos a metas; e (d) execução efetiva de atividades dirigidas a metas.

Pode-se observar que a montagem de um procedimento de avaliação das funções executivas depende da perspectiva teórica adotada pelo profissional. Sendo assim, torna-se importante apresentar brevemente alguns desses modelos teóricos. Burgess e Simons (2005) classificaram as diferentes teorias sobre funções executivas em teorias de sistema único, teorias baseadas em construtos, teorias de processamento múltiplo e teorias de sintoma único.

As teorias que consideram as FE como um sistema único (por exemplo, Cohen \& Servan-Schreiber, 1992; Grafman, 2002) seriam aquelas que propõem que o dano em um único processo ou sistema seria responsável por uma gama de diferentes sintomas disexecutivos. As teorias baseadas em constructos seriam aquelas que se fundamentam em conceitos, como "memória operacional", que seriam conceitos centrais às funções executivas e buscam estruturas cerebrais envolvidas nesses processos. No entanto, ambos os tipos de teorias apresentam limitações (Burgess \& Simons, 2005), por exemplo, estudos utilizando imagens funcionais e medidas eletrofisiológicas sugerem um fracionamento do sistema executivo e, tanto em grupos de pacientes neurológicos quanto em grupos controle, a correlação entre a performance entre tarefas executivas é reduzida. Diante desses achados, pesquisadores começaram a desenvolver modelos mais complexos que consideram que vários componentes diferentes estariam implicados em diversas atividades rotineiras mas poderiam ser observados separadamente em situações experimentais (Fuster, 2002; Stuss \& Alexander, 2002). Outro tipo de modelo consiste das teorias que buscam explicar o funcionamento de uma das funções executivas e o sintoma ligado a sua patologia, estas porém são ainda em menor número. As teorias que buscam os princípios do funcionamento das funções executivas como sistemas complexos tendem a ser em maior número apesar da ausência de consenso sobre qual seria a mais adequada.

\section{Função ou Funções Executivas?}

Evidências advindas da avaliação neuropsicológica (Stuss \& Alexander, 2000; Stuss \& Levine, 2002), da neuroimagem (Carpenter, Just, \& Reichle, 2000; Royall et al., 2002; Smith \& Jonides, 1999) e de pesquisas neurofisiológicas (Funashachi, 2001) têm sugerido a necessidade atual de fracionamento das FE, diferenciando o produto do mecanismo de execução. Essas evidências têm-se fundamentado na observação de que lesões em diferentes regiões do córtex ocasionam diferentes sín- 
dromes neuropsicológicas (Benson \& Miller, 1997; Cummings, 1995; Estévez-Gonzáles et al., 2000; Faw, 2003; Tekin \& Cummings, 2002). Por essas razões, há necessidade de cuidados especiais na avaliação neuropsicológica das FE, pois as relações entre estrutura e função, sistemas e processos, lesão e comprometimento funcional e entre fisiologia e função cognitiva ainda não estão plenamente estabelecidas (Royall et al., 2002; Stuss \& Levine, 2002; Tirapu-Ustárroz et al., 2002).

As evidências na literatura apontam para o fato de que as FE envolvem uma ampla gama de funções cognitivas, em diferentes quadros patológicos, impossíveis de serem avaliadas por uma única prova neuropsicológica. Para este fim, uma distinção básica na análise das FE diz respeito ao elemento que coordena as demais funções (o controle executivo ou sistema supervisor) e as outras funções cognitivas. Em outras palavras, as FE podem ser compreendidas como termo amplo que se refere ao produto de uma operação constituída por vários processos cognitivos para realizar uma tarefa particular, como por exemplo, o raciocínio, a abstração ou o comportamento social. Por sua vez, o controle executivo pode ser compreendido como um sistema ou mecanismo responsável pela coordenação desses processos cognitivos. $\mathrm{Na}$ literatura, muitas vezes, estes termos são tidos como sinônimos (Royall et al., 2002), porém não se tratam de módulos cognitivos idênticos, distintos, antagônicos ou mutuamente excludentes, mas de unidades de análise diferenciadas. Esta consideração é importante porque as evidências (da neuroimagem, da neurofisiologia e da neuropsicologia) sugerem que existem diferentes processos cognitivos envolvidos nas FE, que estão relacionados a diferentes regiões corticais e subcorticais. Em outras palavras, não há uma função executiva unitária ou "homunculus" frontal (Stuss \& Alexander, 2000). Portanto, essa diferenciação entre controle geral e funções cognitivas relacionadas é importante porque auxilia na delimitação de um problema conceitual e possibilita operacionalização na investigação dessas funções. Para uma maior compreensão dessas funções faz-se imprescindível analisar como estas se desenvolvem.

\section{Desenvolvimento e Funções Executivas}

Tal como outras funções, as FE também sofrem um processo de maturação que é multideterminado. Romine e Reynolds (2005) fizeram um estudo meta-analítico sobre o desenvolvimento das funções executivas utilizando-se de pesquisas publicadas entre 1984-2004 e formularam um modelo de desenvolvimento onde as FE só alcançariam sua potencialidade máxima no inicio da vida adulta. Outros estudos sugerem que o desenvolvimento das FE influenciam a regulação emocional e diversas funções cognitivas, o que justificaria a necessidade da criação de um modelo integrado de desenvolvimento emocional e cognitivo (Blair, 2006). O desenvolvimento das FE também parece apresentar papel importante para a aprendizagem de diferentes conteúdos acadêmicos. Vários autores estudaram a relação entre distúrbios de aprendizagem e FE.

As evidências que o processo de envelhecimento também leva a alterações nas FE vem se acumulando na última década. Treitz, Heyder e Daum (2007) estudaram pessoas de 20 a 75 anos de idade e encontraram um declínio significativo em atividade que demandava a inibição de respostas e atenção dividida. MacPherson, Phillips e Della Sala (2002) sugeriram que o declínio das FE deve-se a deterioração no córtex frontal na região dorso-lateral especificamente e não a uma deterioração global. Conseqüentemente, a idade da pessoa a ser avaliada deve ser cuidadosamente considerada tanto durante a escolha dos procedimentos a serem utilizados quanto durante a integração dos resultados.

\section{Instrumentos de Avaliação das Funções Executivas}

A avaliação neuropsicológica é um procedimento de investigação das relações entre cérebro e comportamento, especialmente, das disfunções cognitivas associadas aos distúrbios do Sistema Nervoso Central (Spreen \& Strauss, 1998). A importância da avaliação neuropsicológica está centrada na identificação de pessoas com risco aumentado para desenvolver doenças neurais e no estabelecimento de padrões de desempenho normal. Os instrumentos utilizados na avaliação neuropsicológica são as entrevistas, observações e os testes psicológicos, que auxiliam no diagnóstico clínico, no conhecimento acerca do perfil cognitivo do paciente assim como na estimativa da evolução, prognóstico, delineamento de programas de reabilitação cognitiva e o acompanhamento do tratamento farmacológico e psicossocial. A Tabela 1 apresenta um resumo dos principais testes neuropsicológicos empregados nas avaliações das FE.

Uma preocupação que vem sendo cada dia mais presente, tanto na elaboração de testes quanto na escolha dos mesmos, é a necessidade de obter-se resultados ecologicamente válidos, ou seja, resultados que tenham uma relação clara com o comportamento da pessoa avaliada em situações naturais e que propiciem recomendações pertinentes para o desenvolvimento de planos de reabilitação (Wilson, 1993). Outro ponto importante de ser ressaltado é que pessoas com comprometimento nas FE, freqüentemente, não demonstram rebaixamento em medidas de quociente intelectual (como o QI total fornecido pelas baterias Weschler) nem em medidas tradicionais de funções cognitivas (como memória auditiva e visual), fato que pode levar a resultados que dificultam a detecção de tais comprometimentos.

O Wisconsin Card Sorting Test (WCST) tem sido considerado o padrão-ouro para a avaliação das FE. O WCST consiste num conjunto de 128 cartões com três características distintas: cor (amarelo, verde, vermelho e azul); figuras (círculo, estrela, triângulo e cruz) e núme- 
Hamdan, A. C. \& Pereira, A. P. A. (2009). Avaliação Neuropsicológica das Funções Executivas: Considerações Metodológicas.

Tabela 1

Testes Tradicionais Empregados na Avaliação das Funções Executivas

\begin{tabular}{ll}
\hline Teste & Função cognitiva \\
\hline Wisconsin Card Sorting Test & - Formação de conceitos e solução de problemas, \\
& flexibilidade mental, abstração-raciocínio \\
Trail Making Test & - Flexibilidade mental \\
Teste de Raven & - Abstração-raciocínio \\
Compreensão de provérbios & - Abstração-raciocínio \\
Torre de Londres & - Planejamento \\
Controled Word test & - Fluência verbal \\
Five-Point Test & - Fluência de desenhos \\
Califórnia Verbal learning Test & - Memória \\
Go-No go & - Modulação-inibição de resposta \\
Stroop & - Modulação-inibição de resposta \\
Behavioral Assessment of Dyssecutive Sistem & - Problemas cotidianos por transtornos executivos \\
\hline
\end{tabular}

Nota. Fonte: Porto, 2003; Royall et al., 2002; Spreen \& Strauss, 1998.

ro de figuras (de 1 a 4). A tarefa consiste em associar o conjunto de cartões (divididos em dois grupos de 64) a quatro cartões-estímulo, de acordo com a regra determinada pelo examinador (cor, figura ou número de figuras). Após dez consecutivas associações corretas, as regras são alteradas. O sujeito para ser bem-sucedido necessita deduzir a regra e empregá-la corretamente. A pontuação é realizada de diferentes maneiras, destacando o número de categorias completadas, o número total de erros, o número total de erros perseverados (Spreen \& Strauss, 1998).

Stuss et al. (2000), analisando o desempenho no WCST, principalmente em relação aos erros de perseveração, em pacientes com lesões frontais, encontraram evidências de comprometimento da região dorsolateral. Esta constatação tem sido validada por inúmeros estudos de neuroimagem (Royall et al., 2002), que indicaram ativação das regiões do córtex pré-frontal dorsolateral, particularmente, do lado esquerdo, e de outras regiões cerebrais (pré-frontal anterior direita, menos extensivamente, o cíngulo anterior/mesiofrontal e regiões orbitofrontais). A principal restrição ao WCST está no fato de que lesões nas regiões corticais posteriores também podem afetar o desempenho do teste (Royall et al., 2002).

Estudos recentes têm evidenciado que o comprometimento das FE ocorre não apenas em pacientes com lesões frontais, mas também em pacientes com lesões do tálamo (Van der Werf, Witter, Uylings, \& Jolles, 2000), nas demências degenerativas (Crawford, Blacmore, Lamb, \& Simpson, 2000; Green, 2000), no comportamento antisocial (Brower \& Price, 2001), na dislexia (Brosnan et al., 2002) ou como resultado do processo de alteração normal do envelhecimento (West, 1996; Woodruff-Pak, 1999). As extensas conexões do córtex frontal com outras regiões corticais e subcorticais impõem obstáculos que dificultam as pesquisas clínicas das FE nos diferentes quadros neuropsiquiátricos (Royall et al., 2002).

\section{Implicações para Reabilitação}

Os sintomas de comprometimento das FE mais freqüentemente relatados são dificuldades de planejamento, falta de insight, apatia, perseveração, agitação, distratibilidade, capacidade de tomar decisões limitada e despreocupação com as normas sociais. Diversos estudos encontraram problemas nas FE em pessoas que apresentam uma variedade de patologias do âmbito neurológico e psiquiátrico (Benedict, Dobraski, \& Goldstein, 1999; Chamberlain, Blackwell, Fineberg, Robbins, \& Sahakian, 2005; Oram, Geffen, Geffen, Kavanagh, \& McGrath, 2005). Sendo assim, pesquisas sobre os procedimentos de reabilitação neuropsicológica mais adequado para cada uma das situações vem sendo intensamente propostas.

Dawnson e Guare (2004) sugeriram uma série de diretrizes para intervenções com crianças e adolescentes com déficit em FE, como planejamento, organização e inibição de respostas, utilizando sempre de estratégias de intervenção direcionadas a pessoa e ao ambiente. Esta perspectiva é interessante, pois pode ser aplicada nos diferentes ambientes naturais das crianças e adolescentes, portanto, gerando resultados não apenas nas sessões individuais de consultório, mas facilitando sua incorporação aos hábitos rotineiros dos clientes.

Carney et al. (1999) realizaram uma extensa revisão bibliográfica buscando determinar quais as práticas mais eficazes em reabilitação cognitiva para pessoas com traumatismo crânio encefálico. No entanto, diante do número reduzido das amostras, das definições diferentes utilizadas pelos estudos e pela variedade dos instrumentos utilizados para avaliar as funções antes e depois das intervenções os resultados foram inconclusivos. Estas dificuldades também eram encontradas para determinar procedimentos de reabilitação neuropsicológica para outros grupos. 
Recentemente, Cicerone et al. (2000), publicaram resultados de uma força tarefa que sugeriu princípios de reabilitação e listou alguns procedimentos específicos para serem utilizados diante de déficits funcionais específicos. Neste trabalho, as diretrizes para a reabilitação das FE delineiam a prática de treinamento formal em resolução de problemas em atividades diárias que motivem o cliente e sua família, principalmente, intervenções cognitivas que promovam estratégias de auto-regulação através do uso de auto-instruções verbais, auto-questionamento e automonitoramento. Novamente, a importância de considerar-se variáveis ambientais e implementar mudanças quando necessário são recomendadas.

A Federação Européia de Sociedades Neurológicas também criou uma força de trabalho para avaliar as evidências de eficácia clínica de procedimentos de reabilitação cognitiva e organizou diretrizes de intervenção para diferentes sintomas como afasias e apraxias. A abordagem utilizada nestes estudos que discrimina diversas funções e as relaciona com intervenções especificas, parece ser a mais profícua para construir um corpo de conhecimento sobre as práticas eficazes de reabilitação, ao invés de buscar intervenções gerais por grupo de patologias.

A melhoria e a uniformização dos procedimentos de avaliação das FE executivas pode ter um impacto positivo no planejamento e implementação das intervenções. Assim, torna-se necessário à disseminação de baterias abrangentes e ecologicamente válidas.

\section{Considerações Finais}

A avaliação neuropsicológica das FE tem sido respaldada por vários estudos que evidenciam o comprometimento da performance, em diferentes testes, relacionadas às alterações dos lobos frontais. Royall et al. (2002) destacaram quatro dicotomias importantes em relação à avaliação das FE e o substrato biológico: A primeira é a dicotomia lobo frontal versus sistema frontal - há autores que apóiam a idéia de uma localização única das FE. Contudo, evidências indicam que uma lesão cortical frontal pode ser suficiente, mas não é necessariamente a causa do comprometimento executivo. Apesar das correlações entre funções executivas e lesões frontais ainda não serem plenamente compreendidas, evidências sugerem que lesões, numa rede mais ampla (na rede frontalglânglio basal-tálamocortical), são necessárias para o comprometimento das FE.

A segunda dicotomia é a polarização entre estrutura versus função - esta dicotomia é decorrente da anterior. $\mathrm{O}$ fato de que a função cortical frontal pode estar comprometida por lesões subcorticais (ex. doença vascular) na ausência de patologia cortical frontal demonstrável, evidencia que a FE é dependente da integridade do sistema frontal. A terceira dicotomia é entre controle versus processo - é conhecido o fato de que a FE interfere no desempenho de outros domínios neuropsicológicos. Deste modo, alguns testes que são descritos como testes para avaliação de domínios não-executivos (por exemplo, funções visuoespaciais, memória e linguagem) podem ser sensíveis às patologias do sistema frontal porque requerem a participação do controle executivo. Por outro lado, lesões fora do sistema frontal podem interferir no desempenho de testes de FE, na ausência de disfunção executiva, pela interrupção dos processos que estão sob seu controle durante a realização da tarefa. Por isso, na análise do controle executivo, alguns autores enfatizam o planejamento, a geração de hipóteses e a abstração que nas provas neuropsicométricas podem ser atribuídos à "inteligência geral" ou fator "g". Outros enfatizam subgrupos específicos do sistema frontal e como esse fator interage com outros sistemas para produzir e controlar atividades complexas (Obonsawin et al., 2002).

Por último, a dicotomia entre função executiva versus funções executivas - alguns autores sustentam a idéia de um executivo único (Baddeley, 2003); outros, em oposição, de controles múltiplos para operações cognitivas diferentes (Sylvester et al., 2003). As diferentes medidas neuropsicológicas não sustentam a afirmação de um construto executivo único, ou seja, as medidas de funções executivas são de natureza multidimensional, nenhuma medida avalia todos os domínios das FE e a combinação de diferentes medidas pode complementar a análise das FE.

Em outras palavras, vários obstáculos teóricos e metodológicos dificultam as avaliações neuropsicológicas das FE. Stuss e Alexander (2000), resumiram em cinco pontos as principais limitações dos estudos investigativos sobre as FE: (a) os pacientes, avaliados em amostras, nem sempre apresentam lesões frontais; (b) a inexistência de uma delimitação unitária para as FE; (c) a simples distinção entre processos automáticos e processos controlados não explica a complexidade dos mecanismos de controle; (d) a distinção entre tarefas complexas (relacionadas ao lobo frontal) e tarefas simples (relacionadas a outras áreas corticais) também não explica a diferença entre as funções e as distintas áreas corticais; (e) o papel principal do lobo frontal, possivelmente, está relacionado ao comportamento afetivo e emocional, ao desenvolvimento pessoal, ao juízo social e à autoconsciência, aspectos não avaliados nesses estudos.

Tirapu-Ustárroz et al. (2002) apresentam as seguintes recomendações para a avaliação neuropsicológica das FE: (a) os resultados dos testes neuropsicológicos devem ser considerados de modo integrado a um marco teórico-compreensivo; (b) a escolha dos instrumentos de avaliação das FE deve ser baseada na capacidade de fornecer informações com validade ecológica sobre os mecanismos subjacentes aos déficits; (c) a avaliação neuropsicológica deve ser realizada por pessoas especializadas que saibam interpretar os dados em função de um corpo de conhecimentos sobre as relações entre cérebro e comportamento. 
As evidências atuais da neurofisiologia, da neuroimagem e da neuropsicologia clínica, possibilitam estabelecer um modelo específico para as FE. As FE podem ser compreendidas como um sistema ou mecanismo gerenciador, cuja função básica é a de manutenção (função retrospectiva) e de manipulação (função prospectiva) online da informação. Nas provas neuropsicológicas essas funções são mediadas por processos inibitórios que regulam a interferência de novas e antigas informações (Figura1).

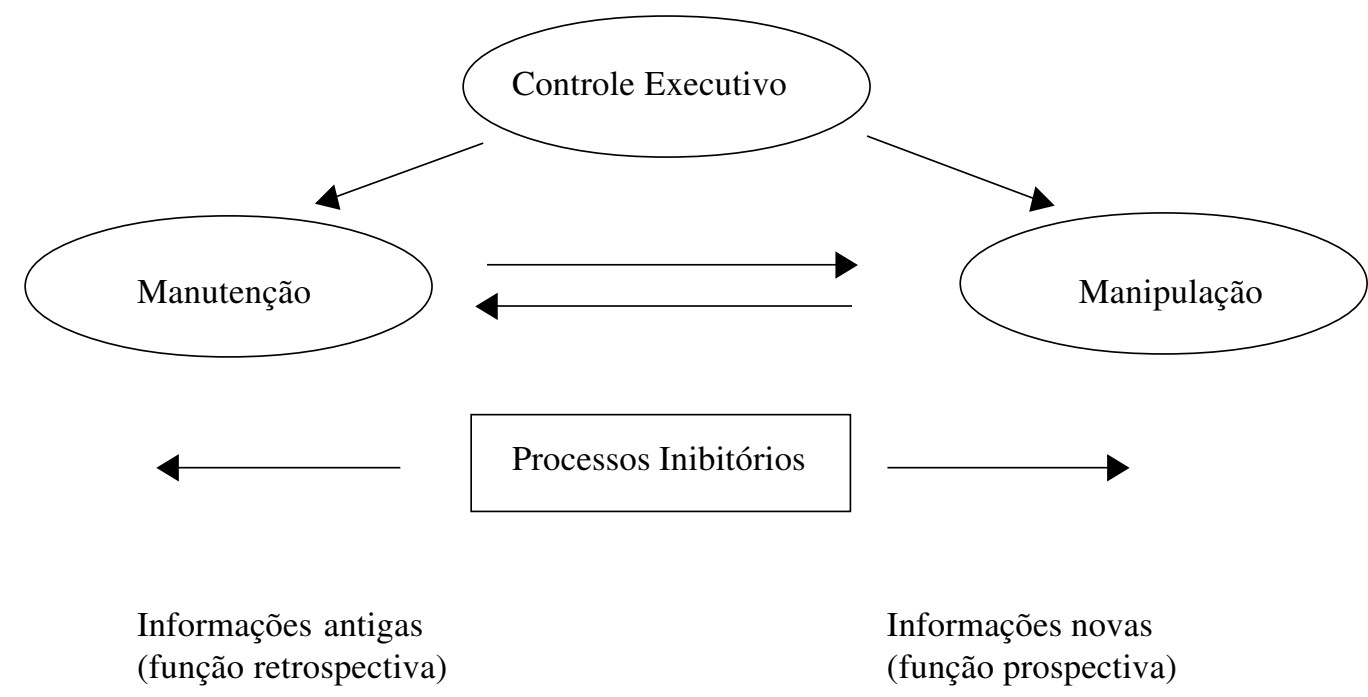

Figura 1. Modelo de funções executivas

Em resumo, o objetivo do presente artigo foi revisar questões metodológicas e conceituais relacionadas à avaliação neuropsicológica das funções executivas. $\mathrm{Na}$ avaliação neuropsicológica, as FE têm sido relacionadas ao planejamento e execução de comportamentos complexos que envolvem muitas funções cognitivas, como atenção, memória, raciocínio e julgamento. As FE, tradicionalmente, estão relacionadas aos lobos frontais.

A organização funcional dos lobos frontais humanos é um dos problemas mais complexos da ciência moderna, e até o presente momento, apenas os primeiros passos foram dados na análise das várias síndromes que podem surgir em casos de lesões frontais e outras partes do cérebro, e isto tem uma importante implicação na avaliação neuropsicológica das FE. As evidências atuais sustentam a hipótese de uma dissociação entre o controle executivo, localizado no Córtex Pré-frontal Dorso Lateral e relacionado a processos cognitivos (memória operativa e atenção), e as demais FE localizadas no Córtex Pré-Frontal Orbital e Córtex Pré-frontal Ventromedial, relacionadas mais à modulação emocional do comportamento.

\section{Referências}

Baddeley, A. (2003). Working memory and language: An overview. Journal Communition Disorder, 36(3), 189-208.

Benedict, R. H. B., Dobraski, M., \& Goldstein, M. Z. (1999). A preliminary study of the association between changes in mood and cognition in a mixed geriatric psychiatry sample. Journals of Gerontology, 54B(2), 94-99.
Benson, D. F., \& Miller, B. L. (1997). Frontal lobes: Clinical and anatomic aspects. In T. D. Freinberg \& M. J. Farah (Eds.), Behavioral neurology and neuropsychology (pp. 401418). New York: McGraw-Hill.

Blair, C. (2006). How similar are fluid cognition and general intelligence? A developmental neuroscience perspective on fluid cognition as an aspect of human cognitive ability. The Behavioral and Brain Sciences, 29(2), 109.

Brosnan, M., Demetre, J., Hamill, S., Robson, K., Shepherd, H., \& Cody, G. (2002). Executive functioning in adults and children with developmental dyslexia. Neuropsychologia, 40(12), 2144-2155.

Brower, M. C., \& Price, B. H. (2001). Neuropsychiatry of frontal lobe dysfunction in violent and criminal behavior: A critical review. Journal of Neurology, Neurosurgery \& Psychiatry, 71, 720-726.

Burgess, P. W., \& Simons, J. S. (2005). Theories of frontal lobe executive function: Clinical applications. In P. W. Halligan \& D. T. Wade (Eds.), Effectiveness of rehabilitation for cognitive deficits (pp. 211-232). Oxford, MA: Oxford University Press.

Carney, N., Chesnut, R. M., Maynard, H., Mann, N. C., Patterson, P., \& Helfand, M. (1999). Effect of cognitive rehabilitation on outcomes for persons with traumatic brain injury: A systematic review. Journal of Head Trauma Rehabilitation, 14(3), 277-307.

Carpenter, P. A., Just, M. A., \& Reichle, E. D. (2000). Working memory and executive function: Evidence from neuroimaging. Current Opinion of Neurobiology, 10(2), 195-199. Chamberlain, S. R., Blackwell, A. D., Fineberg, N. A., Robbins, T. W., \& Sahakian, B. J. (2005). The neuropsychology of obsessive compulsive disorder: The importance of failures in cognitive and behavioral inhibition as candidate endophenotypic markers. Neuroscience and Biobehavioral Reviews, 29, 399-419. 
Cicerone, K. D., Dahlberg, C., Kalmar, K., Langenbahn, D., Malec, J. F., Bergquist, T. F., et al. (2000). Evidence-based cognitive rehabilitation: Recommendations for clinical practice. Archives of Physical and Medical Rehabilitation, 81, 1596-1615.

Cohen, J. D., \& Servan-Schreiber, D. (1992). Context, cortex and dopamine: A connectionist approach to behavior and biology in schizophrenia. Psychological Review, 99, 45-77.

Crawford, J. R., Blacmore, L. M., Lamb, A. E., \& Simpson, S. A. (2000). Is there a differential deficit in fronto-executive functioning in Huntington's Disease? Clinical Neuropsychological Assessment, 1, 4-20.

Cummings, J. F. (1995). Anatomic and behavioral aspects of frontal-subcortical circuits. In K. Grafman, K. J. Holyoak, $\&$ F. Boller, F. (Eds.), Structure and functions of the human prefrontal cortex. Annals of the New York Academy of Sciences, 769, 1-13.

Damásio, A. R. (1996). O erro de Descartes: Emoção, razão e cérebro humano. São Paulo, SP: Companhia das Letras.

Dawnson, P., \& Guare, R. (2004). Executive skills in children and adolescents: A practical guide to assessment and intervention. New York: Guilford Press.

Estévez-Gonzáles, A., Garcia-Sánchez, C., \& Barraquer-Boras, L. I. (2000). Los lóbulos frontales: el cérebro ejecutivo. Revista de Neurología, 31(6), 566-577.

Faw, B. (2003). Pre-frontal executive committee for perception, working memory, attention, long-term memory, motor control, and thinking: A tutorial review. Consciouness and Cognition, 12, 83-139.

Fogel, B. S., Schiffer, R. B., \& Rao, S. M. (Eds.). (2000). Synopsis of neuropsychiatry. Baltimore, PA: Lippincott Williams \& Wilkins.

Freinberg, T. D., \& Farah, M. J. (1997). The development of modern behavioral neurology and neuropsychology. In T. D. Freinberg \& M. J. Farah (Eds.), Behavioral neurology and neuropsychology. New York: McGraw-Hill.

Funahashi, S. (2001). Neuronal mechanisms of executive control by the prefrontal cortex. Neuroscience Research, 39 , 47-165.

Fuster, J. M. (2002). Physiology of executive function: The perception-action cycle. In D. T. Stuss \& R. K. Knight (Eds.), Principles of frontal lobe function (pp. 96-108). New York: Oxford University Press.

Gazzaniga, M., Irvy, R. B., \& Mangun, G. R. (1998). Cognitive neuroscience. The biology of the mind. New York: WW Norton.

Goldberg, E. (2002). O cérebro executivo: Lobos frontais e a mente civilizada. Rio de Janeiro, RJ: Imago.

Grafman, J. (2002). The structured event complex and the human prefrontal cortex. In D. T. Stuss \& R. K. Knight (Eds.), Principles of frontal lobe function (pp. 292-310). New York: Oxford University Press.

Grafman, K., Holyoak, K. J., \& Boller, F. (Eds.). (1995). Structure and functions of the human prefrontal cortex. Annals of the New York Academy of Sciences, 769.

Green, J. (2000). Neuropsychological evaluation of the older adult: A clinician's guidebook. San Diego, CA: Academic Press.

Lezak, M. D. (1995). Neuropsychology assessment (3nd ed.). New York: Oxford University Press.
Loring, D. (Ed.). (1999). INS Dictionary of neuropsychology. New York: Oxford University Press.

Luria, A. R. (1981). Fundamentos de neuropsicologia. São Paulo, SP: Editora da Universidade de São Paulo.

MacPherson, S. E., Phillips, L. H., \& Della Sala, S. (2002). Age, executive function, and social decision making: A dorsolateral prefrontal theory of cognitive aging. Psychology and Aging, 17(4), 598-609.

Obonsawin, M. C., Crawford, J. R., Page, J., Chalmers, P., Cochane, R., \& Low, G. (2002). Performance on tests of frontal lobe function reflect general intellectual ability. Neuropsychologia, 40, 970-977.

Oram, J., Geffen, G. M., Geffen, L. B., Kavanagh, D. J., \& McGrath, J. J. (2005). Executive control of working memory in schizophrenia. Psychiatry Research, 135, 81-90.

Porto, C. S. (2003). Síndromes frontais: Avaliação neuropsicológica. In R. Nitrini, P. Caranelli, \& L. L. Mansur (Eds.), Neuropsicologia: Das bases anatômicas à reabilitação (pp. 73-87). São Paulo, SP: Editora da Universidade de São Paulo.

Romine, C. B., \& Reynolds, C. R. (2005). A model of the development of frontal lobe functioning: Findings from a meta-analysis. Applied Neuropsychology, 12, 190-201.

Royall, D. R., Lauterbach, E. C., Cummings, J. L., Reeve, A., Rummans, T. A., Kaufer, D. I., et al. (2002). Executive control function: A review of its promise and challenges for clinical research. A report from the Committee on Research of the American Neuropsychiatric Associations. Journal of Neuropsychiatry Clinical Neuroscience, 14(4), 377-405.

Smith, E. E., \& Jonides J. (1999). Storage and executive processes in the frontal lobes. Science, 283, 1657-1661.

Spreen, O., \& Strauss, E. (1998). A compendium of neuropsychological tests. Administration, norms, and commentary. New York: Oxford University Press.

Stuss, D. T., \& Alexander, M. P. (2000). Executive functions and the frontal lobes: A conceptual view. Psychological Research, 63, 289-298.

Stuss, D. T., \& Alexander, M. P. (2002). Executive functions and the frontal lobes: A conceptual review. Psychological Research, 63, 289-298.

Stuss, D. T., \& Levine, B. (2002). Adult clinical neuropsychology: Lessons from studies of the frontal lobes. Annual Review of Psychology, 53, 401-403.

Stuss, D. T., Levine, B., Alexander, M. P., Hong, J., Palumbo, C., Hamer, L., et al. (2000). Wisconsin Card Sorting Test performance in patients with local frontal and posterior brain damage: Effects of lesion and test structure on separable cognitive process. Neuropsychologia, 38, 388-402.

Sylvester, C-Y. C., Wager, T. D., Lacey, S. C., Hernandez, L., Nichols, T. E., Smith, E. E., et al. (2003). Switching attention and resolving interference: fMRI measures of executive functions. Neuropsychologia, 41, 357-370.

Tekin, S., \& Cummings, J. L. (2002). Frontal-subcortical neuronal circuits and clinical neuropsychiatry: An update. Journal of Psychosomatic Research, 53, 647-654.

Tirapu-Ustárroz, J., Muñoz-Céspedes, J. M., \& PelegrínValero, C. (2002). Funciones ejecutivas: necessidad de uma integración conceptual. Revista de Neurología, 34, 673-85. 
Hamdan, A. C. \& Pereira, A. P. A. (2009). Avaliação Neuropsicológica das Funções Executivas: Considerações Metodológicas.

Treitz, F. H., Heyder, K., \& Daum, I. (2007). Differential course of executive control changes during normal aging. Neuropsychology, Development, and Cognition, 14B(4), 370-393.

Van Der Werf, Y, D., Witter, M. P., Uylings, H. B. M., \& Jolles, J. (2000). Neuropsychology of infarctions in the thalamus: A review. Neuropsychologia, 38, 613-627.

West, R. L. (1996). An application of prefrontal cortex function Theory to Cognitive Aging. Psychological Bulletin, 120(2), 272-292.

Wilson, B. A. (1993). Ecological validity of neuropsychological assessment: Do neuropsychological indexes predict performance in everday activities. Applied \& Preventive Psychology, 2(4), 209-215.

Woodruff-Pak, D. (1999). The neuropsychology of aging. Oxford, UK: Blackwell. 